\title{
The Geometry of the Mappings by General Dirichlet Series
}

\author{
Dorin Ghisa \\ York University, Glendon College, Toronto, Canada \\ Email:dghisa@yorku.ca
}

How to cite this paper: Ghisa, D. (2017) The Geometry of the Mappings by General Dirichlet Series. Advances in Pure Mathematics, 7, 1-20.

http://dx.doi.org/10.4236/apm.2017.71001

Received: November 22, 2016

Accepted: January 19, 2017

Published: January 23, 2017

Copyright (C) 2017 by author and Scientific Research Publishing Inc. This work is licensed under the Creative Commons Attribution International License (CC BY 4.0).

http://creativecommons.org/licenses/by/4.0/

\begin{abstract}
We dealt in a series of previous publications with some geometric aspects of the mappings by functions obtained as analytic continuations to the whole complex plane of general Dirichlet series. Pictures illustrating those aspects contain a lot of other information which has been waiting for a rigorous proof. Such a task is partially fulfilled in this paper, where we succeeded among other things, to prove a theorem about general Dirichlet series having as corollary the Speiser's theorem. We have also proved that those functions do not possess multiple zeros of order higher than 2 and the double zeros have very particular locations. Moreover, their derivatives have only simple zeros. With these results at hand, we revisited GRH for a simplified proof.
\end{abstract}

\section{Keywords}

General Dirichlet Series, $S_{k}$ Strips, Intertwining Curves,

Fundamental Domains, Riemann Hypothesis

\section{Introduction}

The study of general Dirichlet series has its origins in the works [1]-[18] of E. Cahen, J. Hadamard, E. Landau, H. Bohr, G. H. Hardi, M. Riesz, T. Kojima, M. Kuniyeda, G. Valiron, etc. A lot of contemporary mathematicians created a diversified theory of general Dirichlet series, some insisting on the connection with the Laplace-Stieltjes transforms [19]-[24] as J. Yu, Y. Kong, S. Daochun, X. Luo, Y. Yan, C. Singhal, G. S. Srivastava, etc., others as P.K. Kamthan, S. K. Shing Gautam, L.H. Khoi, [25] [26] endowing them with some topological structures, extending to them the Nevanlinna theory (too many to be cited) or dealing with vector valued Dirichlet series [27]-[32] as A. Defant, M. Maestre, D. Perez-Garcia, J. Bonet, B.L. Srivastava, A. Sharma, G. S. Srivastava, etc.

We will use normalized series defined as follows. To any sequence 
$A=\left(1=a_{1}, a_{2}, \cdots\right)$ of complex numbers and any increasing sequence $\Lambda=\left(0=\lambda_{1}<\lambda_{2}<\cdots\right)$ such that $\lim _{n \rightarrow \infty} \lambda_{n}=\infty$ we associate the series

$$
\zeta_{A, \Lambda}(s)=\sum_{n=1}^{\infty} a_{n} \mathrm{e}^{-\lambda_{n} s}
$$

$\Lambda$ is called the type of the series (1) and the series defined by the same $\Lambda$ will be called series of the same type. When $\lambda_{n}=\ln n$, we obtain the ordinary (proprement dites, [18]) Dirichlet series.

Suppose that $A$ and $\Lambda$ are such that the abscissa of convergence (see [14] [18]) of the series (1)

$$
\sigma_{c}=\limsup _{n \rightarrow \infty} \frac{1}{\lambda_{n}} \log \left|\sum_{k=1}^{n} a_{k}\right| \text { is finite. }
$$

Then (see [1]) $z=\zeta_{A, \Lambda}(s)$ is an analytic function in the half plane $\mathfrak{R}(s)>\sigma_{u}$, where $\sigma_{u}$ is the abscissa of uniform convergence of (1), and where $\sigma_{u}$ is at most $\sigma_{c}+D$ with

$$
D=\limsup _{n \rightarrow \infty} \frac{\log n}{\lambda_{n}}
$$

For the ordinary Dirichlet series $D=1$ and it is known that in the case of Dirichlet L-series defined by imprimitive Dirichlet characters $\sigma_{c}=0$ and $\sigma_{u}=1$, while in the case of primitive characters $\sigma_{c}=\sigma_{u}=0$.

In general, when $D=0$ then $\sigma_{u}=\sigma_{c}$, and the series (1) is an analytic function in the half plane $\mathfrak{R}(s)>\sigma_{c}$.

Suppose that this function can be continued analytically to the whole complex plane, except possible at $s=1$ which is a simple pole. We keep the notation $\zeta_{A, \Lambda}(s)$ for the function obtained by analytic continuation.

With the exception of a discrete set of points from the complex plane, this function is locally injective, i.e. it maps conformally and hence bijectively small neighbourhoods of every point onto some domains. Enlarging these neighbourhoods, the image domains get bigger. How big can they get? The answer is: they become the whole complex plane with some slits (see [33] [34] [35] [36]). A region with this property is called fundamental domain of $\zeta_{A, \Lambda}(s)$.

Our aim is to show that the complex plane can be divided into a countable number of sets whose interiors are fundamental domains of this function. We have done this previously for the Riemann Zeta function [37] [38] as well as for Dirichlet L-functions [35]. To do the same thing for functions obtained by analytic continuations of general Dirichlet series we made [36] the assumption that (1) satisfies a Riemann type of functional equation. We will show next that such a strong assumption is not necessary and we can obtain the same result by using just elementary properties of the conformal mapping.

It has been proved [36] that for normalized series (1) the limit $\lim _{\sigma \rightarrow+\infty} \zeta_{A, \Lambda}(\sigma+i t)=1$ is uniform with respect to $t$. This means that for any $\epsilon>0$ there is $\sigma_{\epsilon} \geq \sigma_{c}$ such that $\sigma>\sigma_{\epsilon}$ implies $\left|\zeta_{A, \Lambda}(s)-1\right|<\epsilon$, therefore the whole half plane $\mathfrak{R}(s)>\sigma_{\epsilon}$ is mapped by $\zeta_{A, \Lambda}(s)$ into a disc centred at $z=1$ of radius $\epsilon$. 
An immediate consequence of this fact is that the abscissa of convergence of a normalized series is less than $+\infty$. Moreover, for $\epsilon<1$ there is no zero of $\zeta_{A, \Lambda}(s)$ in the half plane $\mathfrak{R}(s)>\sigma_{\epsilon}$. Also, if $\zeta_{A, \Lambda}(s)$ satisfies a Riemann type of functional equation, then there cannot be non trivial zeros of $\zeta_{A, \Lambda}(s)$ in the half plane $\mathfrak{R}(s)<-\sigma_{\epsilon}$ neither.

\section{Pre-Images of Lines and Circles}

Suppose that, for a point $s_{0}$ with $\mathfrak{R}\left(s_{0}\right)>\sigma_{c}$ the function $\zeta_{A, \Lambda}(s)$ has a real value $\zeta_{A, \Lambda}\left(s_{0}\right)>1$. The continuation from $s_{0}$ along the interval $[1,+\infty)$, is a curve $\Gamma_{k}^{\prime}$ such that when $z=\zeta_{A, \Lambda}(s)$ tends to 1 , we have that $\sigma$ tends to $+\infty$ on $\Gamma_{k}^{\prime}$, or there is a point $u_{k, j}$ such that $\zeta_{A, \Lambda}\left(u_{k, j}\right)=1$ and the continuation can be carried along the whole real axis giving rise to a curve $\Gamma_{k, j}$. We will show later that when $z=\zeta_{A, \Lambda}(s)$ tends to $+\infty$ on the real axis then $\sigma$ tends to $-\infty$ on $\Gamma_{k}^{\prime}$, or on $\Gamma_{k, j}$, in other words these curves cannot remain in a right half plane.

Let us notice first that $\zeta_{A, \Lambda}(s)$ are transcendental functions and $s=\infty$ is an essential singular point for them. The value $z=0$ cannot be a lacunary value for $\zeta_{A, \Lambda}(s)$ since then $\frac{1}{\zeta_{A, \Lambda}(s)}$ would be a non polynomial integer function for which $s=\infty$ is an essential singular point and hence $s=0$ would be also an essential singular point for $\zeta_{A, \Lambda}(s)$, which is not true. Then, by the Big Picard Theorem, $\zeta_{A, \Lambda}(s)$ has infinitely many zeros in every neighbourhood of $s=\infty$.

Given a bounded region of the plane, there is $r>0$ such that the pre-image of the circle $C_{r}$ centred at the origin and of radius $r$ has only disjoint components, which are closed curves containing each one a unique zero of $\zeta_{A, \Lambda}(s)$ belonging to that region. When $r$ increases those curves expand and they can touch one another at some points $v_{k}$ (see Figure $1(\mathrm{~b})$ ). These are branch points of the function, since in every neighbourhood of $v_{k}$ the function takes at least twice any value on the image circle. Therefore the derivative of $\zeta_{A, \Lambda}(s)$ cancels at $v_{k}$. It is obvious that any zero of the derivative, which is not a zero of the function itself, can be obtained in this way. Indeed, if $v$ is such a zero, we can take $r=\left|\zeta_{A, \Lambda}(v)\right|$ and necessarily at least two components of the pre-image of $C_{r}$ will pass through $v$.

What happens with those components of the pre-image of a circle $C_{r}$ when $r=1$ ? We have proved [36], Theorem 1 that there is at least one unbounded component of the pre-image of the unit circle. That proof did not use the assumption of $\zeta_{A, \Lambda}(s)$ satisfying a Riemann type of functional equation and therefore it is true for any function $\zeta_{A, \Lambda}(s)$.

Let us notice that two curves $\Gamma_{k}^{\prime}$ and $\Gamma_{l}^{\prime}$ cannot intersect each other. Indeed, if $s_{0}$ would be a common point of these curves, then when $z=\zeta_{A, \Lambda}(s)$ moves on the interval $I$ between $z_{0}=\zeta_{A, \Lambda}\left(s_{0}\right)$ and 1 the point $s$ describes an unbounded curve which bounds a domain mapped by $\zeta_{A, \Lambda}(s)$ onto the complex plane with a slit alongside the interval $I$. That domain should contain 


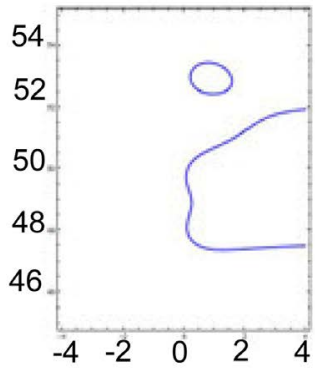

(a)

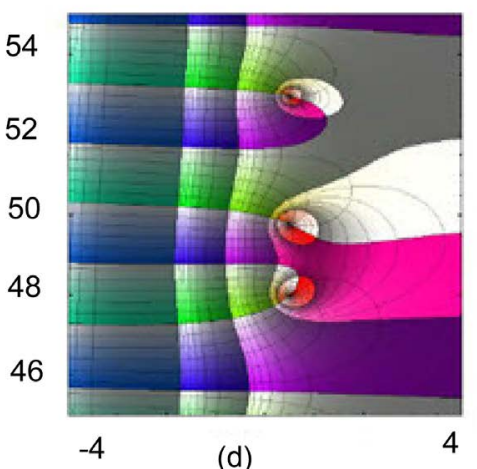

Figure 1. Pre-images of circles and the birth of a strip.

a pole of $\zeta_{A, \Lambda}(s)$ which is not true. Therefore an intersection point $s_{0}$ of the two curves cannot exist and consecutive curves $\Gamma_{k}^{\prime}$ and $\Gamma_{k+1}^{\prime}$ bound infinite strips $S_{k}$. We suppose that $S_{0}$ is the strip containing the point $s=1$ and for every integer $k$, the curve $\Gamma_{k+1}^{\prime}$ is situated above $\Gamma_{k}^{\prime}$. Figure 1(e) illustrates the birth of a strip $S_{k}$ when the pre-image of a ray making a small angle $\alpha$ with the positive real half axis is taken and then we let $\alpha \rightarrow 0$.

We have also proved [36], Theorem 2 that every unbounded component of the pre-image by $\zeta_{A, \Lambda}(s)$ of the unit circle is contained between two consecutive curves $\Gamma_{k}^{\prime}$ and $\Gamma_{k+1}^{\prime}$ and vice-versa, if $k \neq 0$ between two consecutive curves $\Gamma_{k}^{\prime}$ and $\Gamma_{k+1}^{\prime}$ there is a unique unbounded component of the pre-image of the unit circle. It has been shown that the respective component does not intersect any one of these curves. For $k \neq 0$ every strip $S_{k}$ contains also a unique curve $\Gamma_{k, 0}$ which is mapped bijectively by $\zeta_{A, \Lambda}(s)$ onto the interval $(-\infty, 1)$ of the real axis, as well as a certain number of curves $\Gamma_{k, j}$, $j \neq 0$ which are mapped bijectively by $\zeta_{A, \Lambda}(s)$ onto the whole real axis. There are infinitely many strips $S_{k}$ covering the whole complex plane [36], Theorem 4. Therefore, there are infinitely many unbounded components of the pre-image by $\zeta_{A, \Lambda}(s)$ of the unit circle. Some strips $S_{k}$ can contain also bounded components of the pre-image of the unit circle as well as bounded components of the pre-image of $C_{r}$ with $r>1$. Figure 1(d) portrays the strip $S_{5}$ of $\zeta(s)$ containing two components of the pre-image of the unit circle: the unbounded one containing two zeros and the other bounded, containing one zero.

The use of the pre-image of the real axis can be traced back to Speiser's work 
[39] on the Riemann Zeta function. After that the pre-image of the real axis does not appear any more in literature as a tool except for the paper of Arias-de-Reina [40], who revisits Speiser's theorem. In the same year John Derbyshire uses both: the pre-image of the real axis and that of the imaginary axis in his popular book Prime Obsession and declares that they are at the heart of that book. The classification of the components of the pre-image of the real axis by the Riemann Zeta function appears for the first time in [37], where the strips $S_{k}$ are also introduced and a method is devised of partitioning them into fundamental domains. Later, such a classification has been extended to Dirichlet L-functions and finally to functions defined by general Dirichlet series.

A different approach, namely that of phase diagram, has been used by Elias Wegert for visual exploration of complex functions [41] [42]. Applied to the Riemann Zeta function, his phase plots revealed interesting patterns pertaining to the universality property of that function. It is known that such a property extends to more general Dirichlet series and probably it can complement our fundamental domains approach.

An even better way to visualize the complexity of conformal mappings by analytic functions of a complex variable is to use an orthogonal mesh in the z-plane formed with rays issuing from the origin and circles centred at the origin. Moreover, a spectre of colors can be superposed to the mesh as seen in Figures 2(a)-(c). By taking the pre-image of that mesh we obtain a coloured orthogonal mesh in the s-plane in which the color of every point coincides with that of its

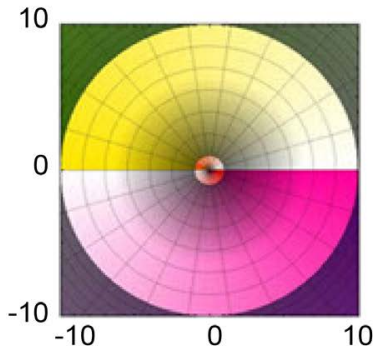

(a)

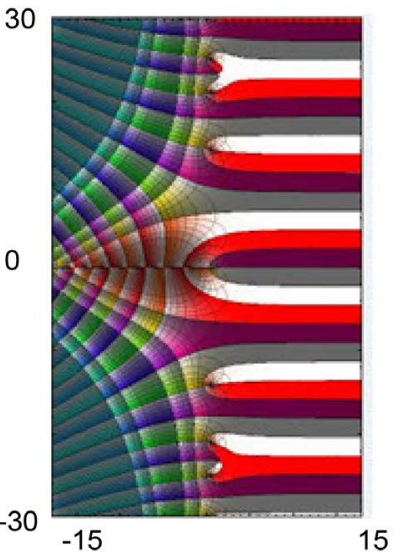

(d)

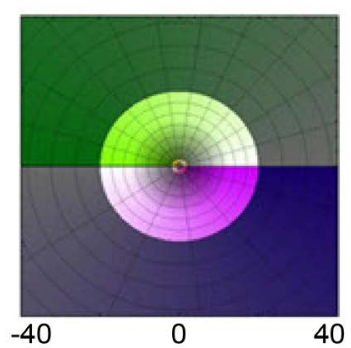

(b)

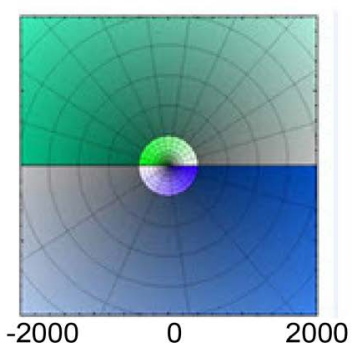

(c)

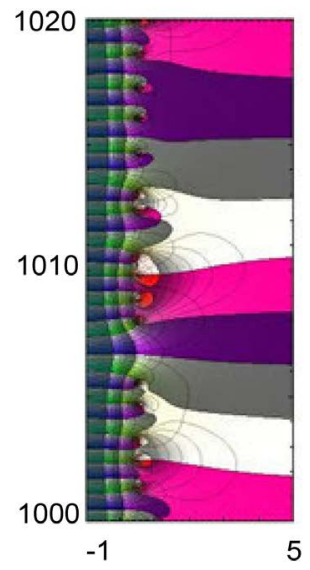

(e)

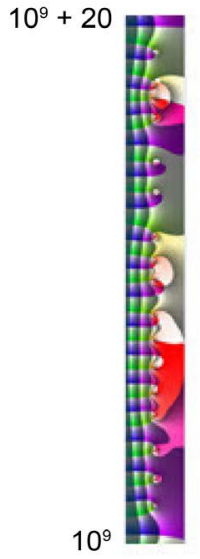

(f)

Figure 2. Colour-visualization of the conformal mapping by $\zeta(s)$. 
image. In this way we can locate the corresponding points in the two planes and have also a global view of the mapping. The pre-image of the real axis and the $S_{k}$-strips are still identifiable. Figures 2 (d)-(f) above illustrates the mapping by the Riemann Zeta function in the rectangles $[-20,20] \times[-30,30]$, $[-1,5] \times[1,000 ; 1,020]$ and $[0.4,1.4] \times\left[10^{9}, 10^{9}+20\right]$. By comparing them, one can notice the increasing number of zeros in the strips with increasing $t$. The pattern we can see here is proper to any function $\zeta_{A, \Lambda}(s)$.

Theorem 1 No zero of $\zeta_{A, \Lambda}(s)$ or of $\zeta_{A, \Lambda}^{\prime}(s)$ can belong to a curve $\Gamma_{k}^{\prime}$.

Proof. The affirmation of the theorem is obvious for the zeros of $\zeta_{A, \Lambda}(s)$ since 0 does not belong to the interval $[1,+\infty)$ of the real axis. A more intricate argument guarantees that the same is true for the zeros of the derivative of $\zeta_{A, \Lambda}(s)$. Indeed, even if $r>1$, no bounded component of the pre-image of $C_{r}$ can reach $\Gamma_{k}^{\prime}$, despite of the fact that $C_{r}$ intersects the interval $[1,+\infty)$. Indeed, in the contrary case, the respective component should intersect $\Gamma_{k}^{\prime}$ at lest twice, or it should be tangent to it, fact which requires that $C_{r}$ intersects the interval $[1,+\infty)$ the same number of times or to be tangent to it, which is not possible. The fact that $C_{r}$ intersects the interval $[1,+\infty)$ has as effect the pre-image of $C_{r}$ intersecting the curves $\Gamma_{k, j}$ (and not $\Gamma_{k}^{\prime}$ ). It results that no two bounded components of the pre-image of $C_{r}$ can meet on $\Gamma_{k}^{\prime}$, neither can one of these components meet on $\Gamma_{k}^{\prime}$ an unbounded component of the pre-image of $C_{r}$ into a zero of $\zeta_{A, \Lambda}^{\prime}(s)$.

Remark: Theorem 1 does not imply that $\zeta_{A, \Lambda}^{\prime}(s)$ cannot have zeros on some $\Gamma_{k, j}$. Such zeros appeared as possible for the Dirichlet L-function $L(5,2, s)$ as seen in Figure 3 when $t$ has approximately the values 169.2 and 179.2. However, we suspected that this was due to the poor resolution of the picture and indeed, when we zoomed on the respective points, we obtained configurations which show clearly that $\zeta_{A, \Lambda}^{\prime}(s)$ does not cancel there. However, as we will see later, the possibility of such zeros cannot be excluded.

We have seen that the unbounded components of the pre-image of the unit circle do not intersect any $\Gamma_{k}^{\prime}$. On the other hand the bounded components of the pre-image of the unit circle intersect curves $\Gamma_{k, j}$ at points $u_{k, j}$ where $\zeta_{A, \Lambda}\left(u_{k, j}\right)={ }_{-}^{+} 1$. In the same way the bounded components of the pre-image of $C_{r}$ with $r>1$ will intersect $\Gamma_{k, j}$ at points $u$ where $\zeta_{A, \Lambda}(u)=_{-}^{+} r$. However the story of the unbounded components of the pre-image of $C_{r}$ is a little more complicated.

When increasing $r$ past 1 all the unbounded components of the pre-image of $C_{1}$ fuse together into a unique unbounded curve $\gamma_{r}$ intersecting every curve $\Gamma_{k}^{\prime}$, hence they do not generate by this fusion zeros of $\zeta_{A, \Lambda}^{\prime}(s)$. Indeed, since the mapping of $\Gamma_{k}^{\prime}$ onto the interval $(1,+\infty)$ is bijective, there should be a unique point $s_{k}$ on every $\Gamma_{k}^{\prime}$ such that $\zeta_{A, \Lambda}\left(s_{k}\right)=r$. The continuation over $C_{r}$ from each one of these points can be made clockwise and counter clockwise into $S_{k}$, respectively $S_{k-1}$, for every $k$, giving rise to that unbounded curve. The final conclusion is that $\zeta_{A, \Lambda}^{\prime}(s)$ does not cancel on any $\Gamma_{k}^{\prime}$. 

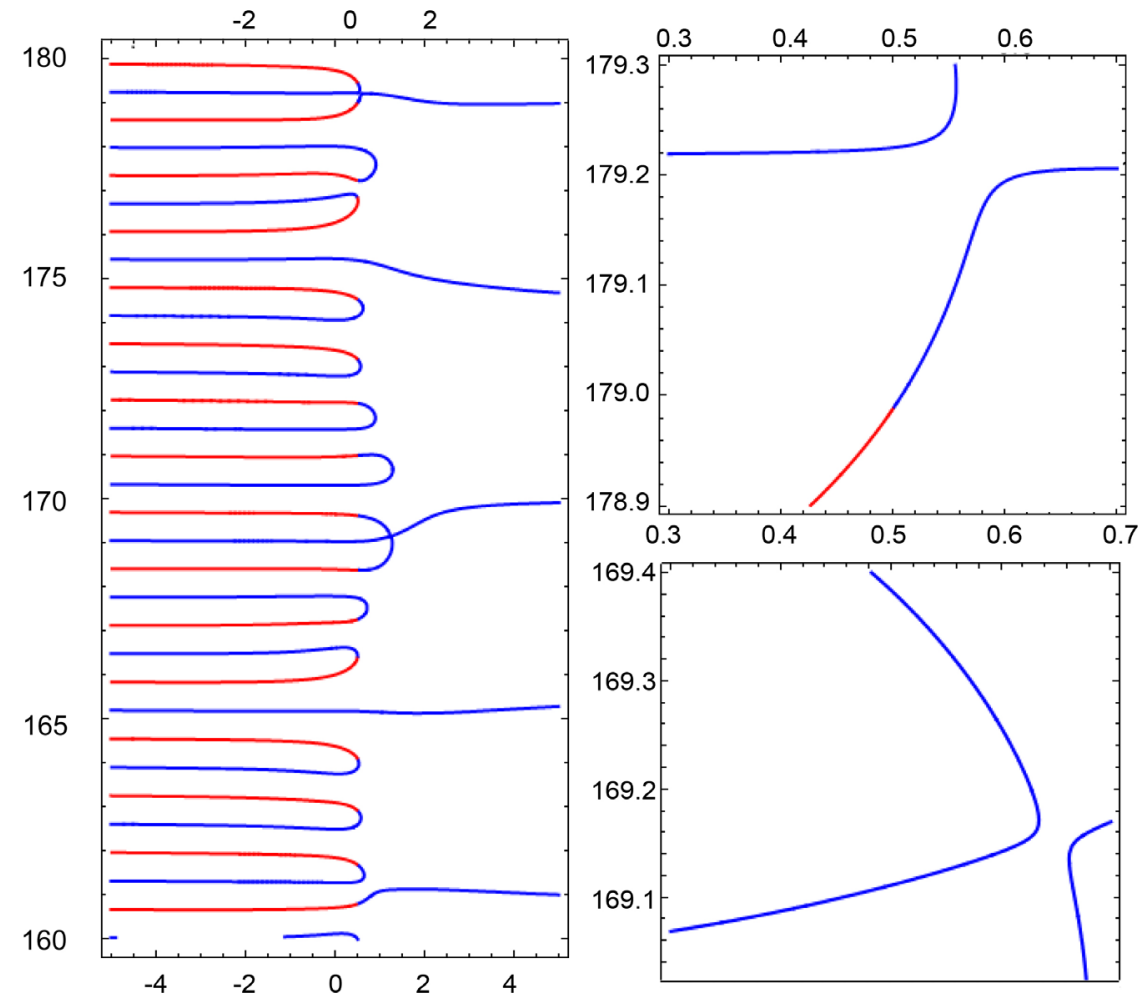

Figure 3. Possible zeros of $\zeta_{A, \Lambda}^{\prime}(s)$ on $\Gamma_{k, j}$ which are not double zeros of $\zeta_{A, \Lambda}(s)$.

Given any bounded domain in the plane $(s)$, we can take $r>1$ close enough to 1 such that $\gamma_{r}$ does not touch any bounded component of the pre-image of $C_{r}$ included in that domain. However, for bigger values of $r$ the curve $\gamma_{r}$ comes into contact with bounded components which were turning around one or several zeros $s_{k, j}$ of $\zeta_{A, \Lambda}(s)$, fusing with them and getting to the left of those zeros, hence intersecting also the curves $\Gamma_{k, j}$ which contain the respective zeros. The curve $\gamma_{r}$ is orthogonal to every component of the preimage of the real axis if it intersects that component at a point where $\zeta_{A, \Lambda}^{\prime}(s)$ does not cancel.

Since a point turning around the origin in the same direction on an arbitrary circle $C_{r}$ centred at the origin will meet consecutively the positive and the negative real half axis, the components of the pre-image of $C_{r}$ (including $\gamma_{r}$ when $r>1$ ) should meet consecutively the pre-image of the positive and the negative half axis (coloured differently). This is [33] [35] the so-called color alternating rule.

An immediate consequence of this rule is that in every strip $S_{k}$ the first and the last curve $\Gamma_{k, j}$ should be such that the pre-image of the negative real half axis faces the corresponding $\Gamma_{k}^{\prime}$. Then, consecutive $\Gamma_{k, j}$ have the same orientation, as long as they are on the same side of $\Gamma_{k, 0}$. A zero $s_{0}$ of $\zeta_{A, \Lambda}^{\prime}(s)$ being on $\Gamma_{k, j}$ and not being a zero of $\zeta_{A, \Lambda}(s)$ (where the colors are changing) implies that different components of the pre-image of a circle $C_{r}$ fuse (for a value $r_{0}$ of $r$ ) on $\Gamma_{k, j}$. The respective components must obey the color alternating rule for every $r<r_{0}$ and we realize that after fusion the component 
can be such that the rule is still in force. Indeed, if $\Gamma_{k, j}$ and $\Gamma_{k, j^{\prime}}$ have the same color at $s_{0}$ then this curve can continue to meet the respective color without affecting the color alternation by simply switching the branches of $\Gamma_{k, j}$ and $\Gamma_{k, j^{\prime}}$ which come to $s_{0}$. However, as we will see next, the position of $s_{0}$ with respect to the two zeros of $\zeta_{A, \Lambda}(s)$ on these curves cannot be arbitrary.

Theorem 2 For $k$ different of 0 , there is no zero of the derivative of $\zeta_{A, \Lambda}^{\prime}(s)$ at the left of the leftmost zero of $\zeta_{A, \Lambda}(s)$ in $S_{k}$

Proof. We can reproduce the proof of Theorem 1 from [43] for arbitrary functions $\zeta_{A, \Lambda}(s)$. Let $s_{k, j}$ be the leftmost zero of $\zeta_{A, \Lambda}(s)$ from $S_{k}$ and suppose that the simple zero $v_{k, j}$ of $\zeta_{A, \Lambda}^{\prime}(s)$ is a progenitor of $s_{k, j}$, i.e. a component of the pre-image of the circle $C_{r}$ with $r=\left|\zeta_{A, \Lambda}\left(v_{k, j}\right)\right|$ contains the point $s_{k, j}$. It is obviously enough to deal with the case where $j>0$, i.e. where $\Gamma_{k, j}$ is above $\Gamma_{k, 0}$. Then $\zeta_{A, \Lambda}\left(v_{k, j}\right)$ (indicated in Figure 4 as $L\left(v_{k, j}\right)$, since it has been partially computer generated by a Dirichlet L-function) belongs to the upper half plane and $0 \leq \alpha<\pi$, where $\alpha=\arg \zeta_{A, \Lambda}\left(v_{k, j}\right)$.

The pre-image of the ray determined by $\zeta_{A, \Lambda}\left(v_{k, j}\right)$ contains two curves which are orthogonal at $v_{k, j}$. The angles at $v_{k, j}$ are doubled by $\zeta_{A, \Lambda}(s)$, hence the four arcs of the pre-image of that ray make angles of $\alpha / 2, \pi / 2+\alpha / 2$, $\pi+\alpha / 2$ and $3 \pi / 2+\alpha / 2$ with a horizontal line whose image passes through $\zeta_{A, \Lambda}\left(v_{k, j}\right)$. The angle $\pi / 2+\alpha / 2$ made by the second arc (which ends in $s_{k, j}$ ) with this line is less than the angle $\beta$ made by the tangent to the pre-image of the respective ray at any point between $s_{k, j}$ and $v_{k, j}$ with the same horizontal line. If $\mathfrak{R}\left(v_{k, j}\right) \leq \mathfrak{R}\left(s_{k, j}\right)$, then there must be a point on that arc for which $\beta=\pi / 2$, therefore $\alpha<0$, which is absurd.

Corollary: If $\zeta_{A, \Lambda}(s)$ satisfies $\mathrm{RH}$, then the zeros of $\zeta_{A, \Lambda}^{\prime}(s)$ from every strip $S_{k}$ are at the right side of the critical line. When $\zeta_{A, \Lambda}(s)$ is the Riemann

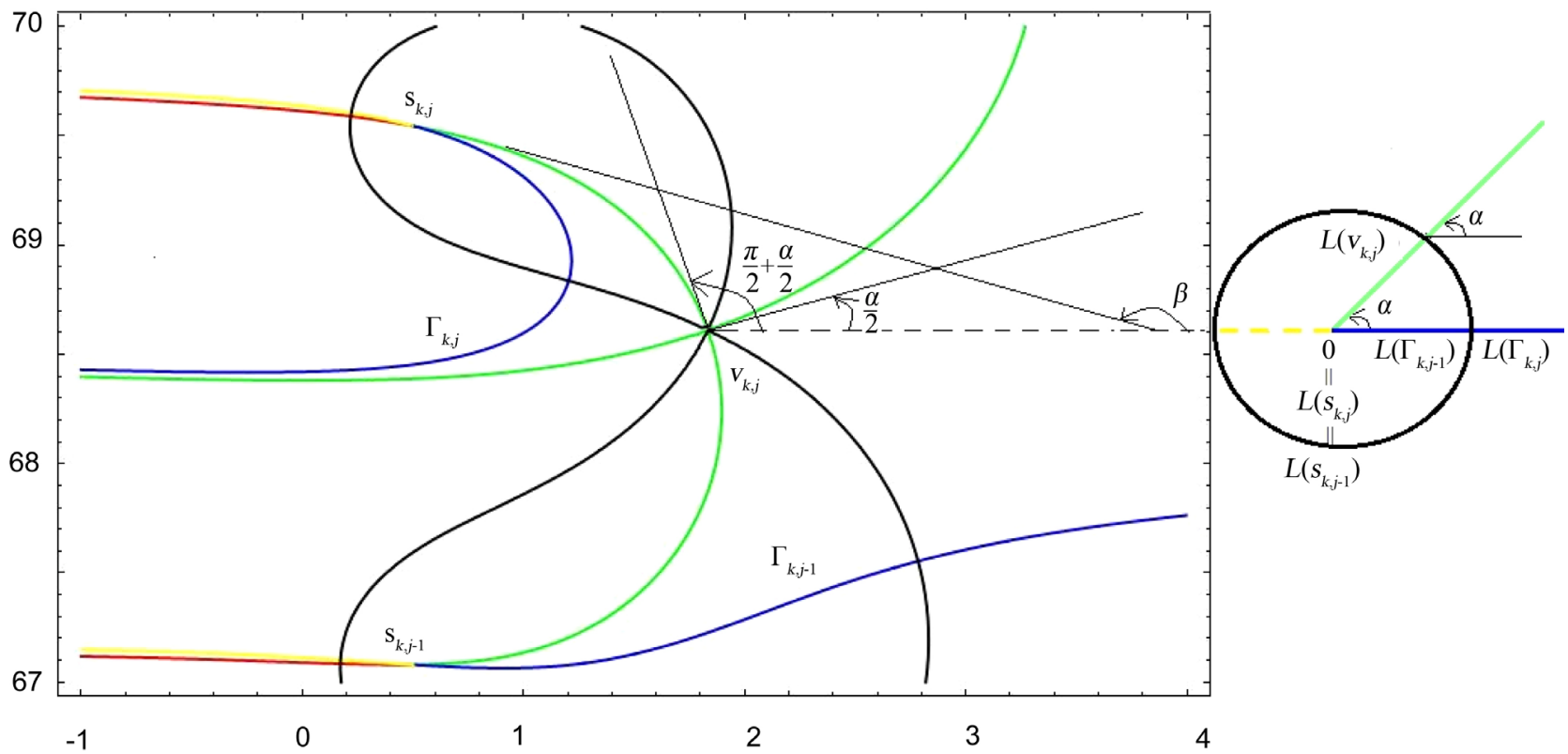

Figure 4. The location of the zeros of $\zeta_{A, \Lambda}^{\prime}(s)$. 
Zeta function this corollary represents the Speiser's theorem [39].

The existence of multiple zeros of functions obtained by analytic continuations of Dirichlet series has been documented (probably for the first time) in [44], where double zeros of a linear combination of Dirichlet L-functions have been found (see Figure 5 below).

We have shown that all those double zeros are located on the critical line. In this example the function is $L(7,2, s)+0.34375 L(7,4, s)$ and the double zero is obtained for the approximate value of $s$ of $0.5+31.6 \mathrm{i}$. The double zeros we have found for all the functions of this type were located at the intersection of $\Gamma_{k, 0}$ and $\Gamma_{k, 1}$ or of $\Gamma_{k, 0}$ and $\Gamma_{k,-1}$. We can make now a much more general affirmation about the multiple zeros of functions obtained by analytic continuation of general Dirichlet series.

Theorem 3 In every strip $S_{k}$ of a function $\zeta_{A, \Lambda}(s)$ this function has at most one double zero. Such a zero is found at the intersection of $\Gamma_{k, 0}$ and $\Gamma_{k, 1}$ or of $\Gamma_{k, 0}$ and $\Gamma_{k,-1}$. There is no multiple zero of $\zeta_{A, \Lambda}^{\prime}(s)$ in $S_{k}$ and hence no zero of a higher order than two of $\zeta_{A, \Lambda}(s)$.

We need to postpone the proof of this theorem for a while.

\section{Intertwining Curves}

When studying functions $z=\zeta_{A, \Lambda}(s)$ it is useful to consider besides the planes $(s)$ and $(z)$ also a plane $(w)$, where $w=\zeta_{A, \Lambda}^{\prime}(s)$. Sometimes the planes $(z)$ and $(w)$ will be identified in order to make more obvious certain relations between the configurations defined by the two functions in the respective planes. The configurations we have in view are pre-images by both $\zeta_{A, \Lambda}(s)$ and by
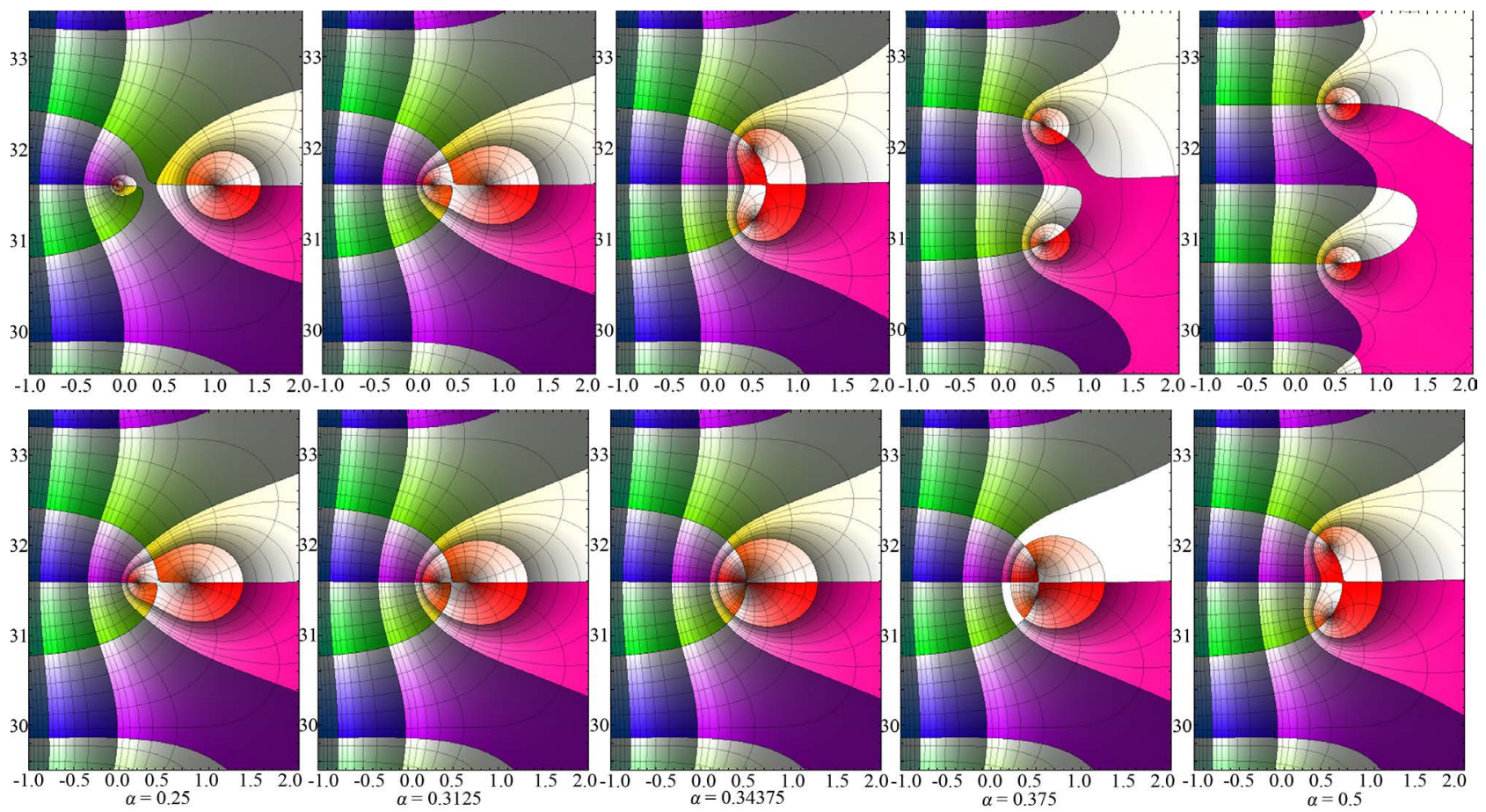

Figure 5. A double zero of a linear combination of dirichlet L-functions. 
$\zeta_{A, \Lambda}^{\prime}(s)$, of some curves or domains.

Regarding the pre-image by $\zeta_{A, \Lambda}(s)$ and by $\zeta_{A, \Lambda}^{\prime}(s)$ of the real axis it has been found [34] [36] that the components of these pre-images are paired in such a way that only the components of the same pair can intersect each other. The respective pairs form the so-called intertwining curves.

Three kinds of intertwining curves have been distinguished [33] [34] [35] [36], namely:

1) $\Gamma_{k}^{\prime}$ and $\Upsilon_{k}^{\prime}, k \in \mathbb{Z}$, which are mapped bijectively by $\zeta_{A, \Lambda}(s)$ and by $\zeta_{A, \Lambda}^{\prime}(s)$ onto the interval $(1,+\infty)$, respectively $(-\infty, 0)$,

2) $\Gamma_{k, 0}$ and $\Upsilon_{k, 0}, k \in \mathbb{Z}$, which are mapped bijectively by $\zeta_{A, \Lambda}(s)$ and by $\zeta_{A, \Lambda}^{\prime}(s)$ onto the interval $(-\infty, 1)$, respectively $(0,+\infty)$

3) $\Gamma_{k, j}$ and $\Upsilon_{k, j}, j \neq 0, k \in \mathbb{Z}, j \in J_{k} \backslash\{0\}=$ (a finite set of integers), which are mapped bijectively by $\zeta_{A, \Lambda}(s)$, respectively by $\zeta_{A, \Lambda}^{\prime}(s)$ onto the whole real axis.

Theorem 4 The intertwining curves touch each other at the points where the tangent to $\Gamma_{k, j}$, respectively $\Gamma_{k}^{\prime}$ is horizontal. Vice-versa, if at a point of such a curve the tangent is horizontal, then a component of the pre-image of the real axis by $\zeta_{A, \Lambda}^{\prime}(s)$ passes also through that point.

Proof. Indeed, suppose that $s=s(x)$ is the equation of a curve $\Gamma_{k}^{\prime}$ or of a curve $\Gamma_{k, j}$ such that $\zeta_{A, \Lambda}(s(x))=x$. Then (see Figure 6 below)

$$
\zeta_{A, \Lambda}^{\prime}(s(x)) s^{\prime}(x)=1 \text {. }
$$
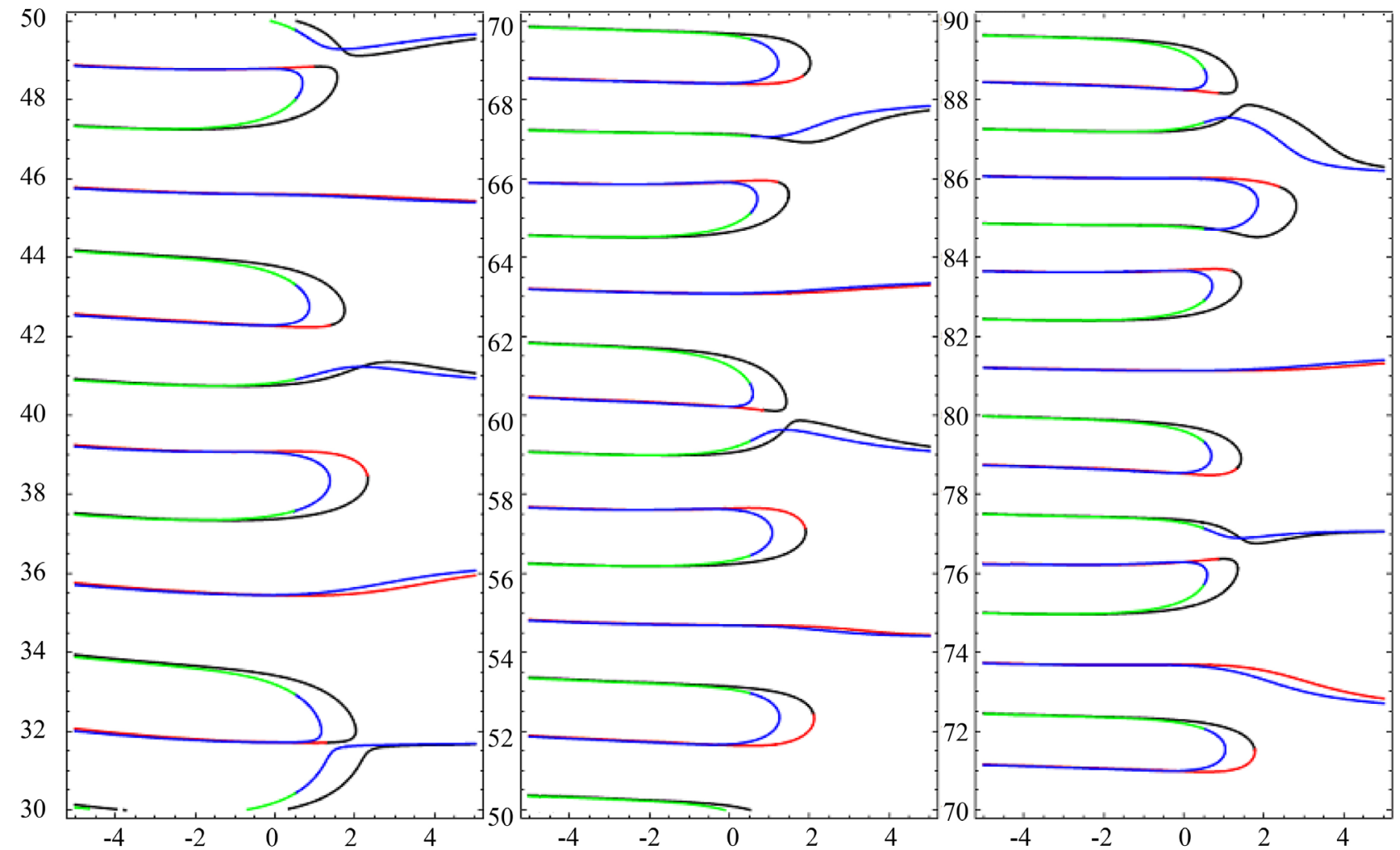

Figure 6. Intertwining curves of $\zeta(\sigma+i t)$ for $t \in[0,100]$. 
The Equation (4) shows that the argument of $\zeta_{A, \Lambda}^{\prime}(s(x))$ is opposite to that of $s^{\prime}(x)$, therefore they cancel simultaneously, and when one is $\pi$, the other should be $-\pi$. Yet these values of the argument of a point mean that the respective point is on the real axis, therefore $s(x)$ belongs to both pre-images of the real axis, by $\zeta_{A, \Lambda}(s)$ and $\zeta_{A, \Lambda}^{\prime}(s)$ which completely proves the theorem.

Remark: The Theorem 4 is a corollary of a much more general property which says that if $z=f(s)$ is an analytic function in a domain $D$ of the complex plane and $\gamma$ is the image by $f(s)$ of a smooth curve $\Gamma: s=s(z)$, then denoting by $\Upsilon$ the pre-image by $f^{\prime}(s)$ of $\gamma$, at every point $s_{0}$ where $\Gamma$ and $\Upsilon$ intersect each other we have $\arg f^{\prime}\left(s_{0}\right)+\arg s^{\prime}\left(z_{0}\right)=0(\bmod 2 \pi)$, where $s_{0}=s\left(z_{0}\right)$.

Indeed, suppose that the planes $(z)$ and $(w)$ are identified, where $w=f^{\prime}(s)$ and write $S=S(w)$ for the curve $\Upsilon$. Then at an intersection point $s_{0}$ of $\Gamma$ and $\Upsilon$ we have $s_{0}=s\left(z_{0}\right)=S\left(f^{\prime}\left(s_{0}\right)\right)$ and $f(s(z))=z$ implies $f^{\prime}(s(z)) s^{\prime}(z)=1$, hence $f^{\prime}\left(s_{0}\right) s^{\prime}\left(z_{0}\right)=1$, etc.

We can show now that:

Theorem 5 No strip $S_{k}$ can be included in a right half plane.

Proof. The formula (4) written for $\Gamma_{k}^{\prime}$ tells us that when $s^{\prime}(x)$ tends to $\infty$ then $\zeta_{A, \Lambda}^{\prime}(s(x))$ must tend to 0 . Yet, there is no zero of $\zeta_{A, \Lambda}^{\prime}(s(x))$ on any $\Gamma_{k}^{\prime}$. On the other hand, $\zeta_{A, \Lambda}^{\prime}(s(x))$ is an unlimited continuation of $\zeta_{A, \Lambda}^{\prime}(s)$ alongside $\Gamma_{k}^{\prime}$, hence when $s(x)$ tends to $\infty$ we have that $\zeta_{A, \Lambda}^{\prime}(s(x))$ tends to $\infty$ not to 0 and this is a contradiction. The conclusion is that the geometry of the pre-image of the real axis is in the whole complex plane similar to that we can see in a bounded region of the plane in Figure 2, Figure 3, Figure 6, Figure 7.

Summarizing these facts and having in view [36] we can say:

Theorem 6 The variable $\sigma$ takes any real value on every curve $\Gamma_{k}^{\prime}$. Consecutive curves $\Gamma_{k}^{\prime}$ form infinite strips $S_{k}$ which are mapped (not necessarily bijectively) onto the whole complex plane with a slit alongside the interval $[1,+\infty)$ of the real axis. If $S_{k}$ contains $m_{k}$ zeros of the function $\zeta_{A, \Lambda}(s)$, then it will contain $m_{k}-1$ zeros of $\zeta_{A, \Lambda}^{\prime}(s)$. For any given $k$, the function $\zeta_{A, \Lambda}(s)$ either has a finite number of zeros $s_{k, j}$ in $S_{k}$, or $\lim _{j \rightarrow \infty} s_{k, j}=\infty$. If $\zeta_{A, \Lambda}(s)$ satisfies a Riemann type of functional equation, every strip $S_{k}, \quad k \neq 0$ contains a finite number of zeros of this function. The strip $S_{0}$ may contain infinitely many zeros of $\zeta_{A, \Lambda}(s)$.

Proof. We only need to justify the numbers $m_{k}$ and $m_{k}-1$ to which the theorem makes reference. We have seen that every zero of $\zeta_{A, \Lambda}^{\prime}(s)$ is obtained when two components of the pre-image of some circle $C_{r}$ centred at the origin and of radius $r$ come into contact. If we consider the zeros of $\zeta_{A, \Lambda}(s)$ as the leafs of a binary tree whose internal nodes are obtained in this way, that tree is a complete binary tree and it is known that it must have exactly $m_{k}-1$ internal nodes. Figure 7 illustrates this situation for ten strips of the Riemann Zeta function. The location of the zeros of $\zeta(s)$ and those of its first two derivative 

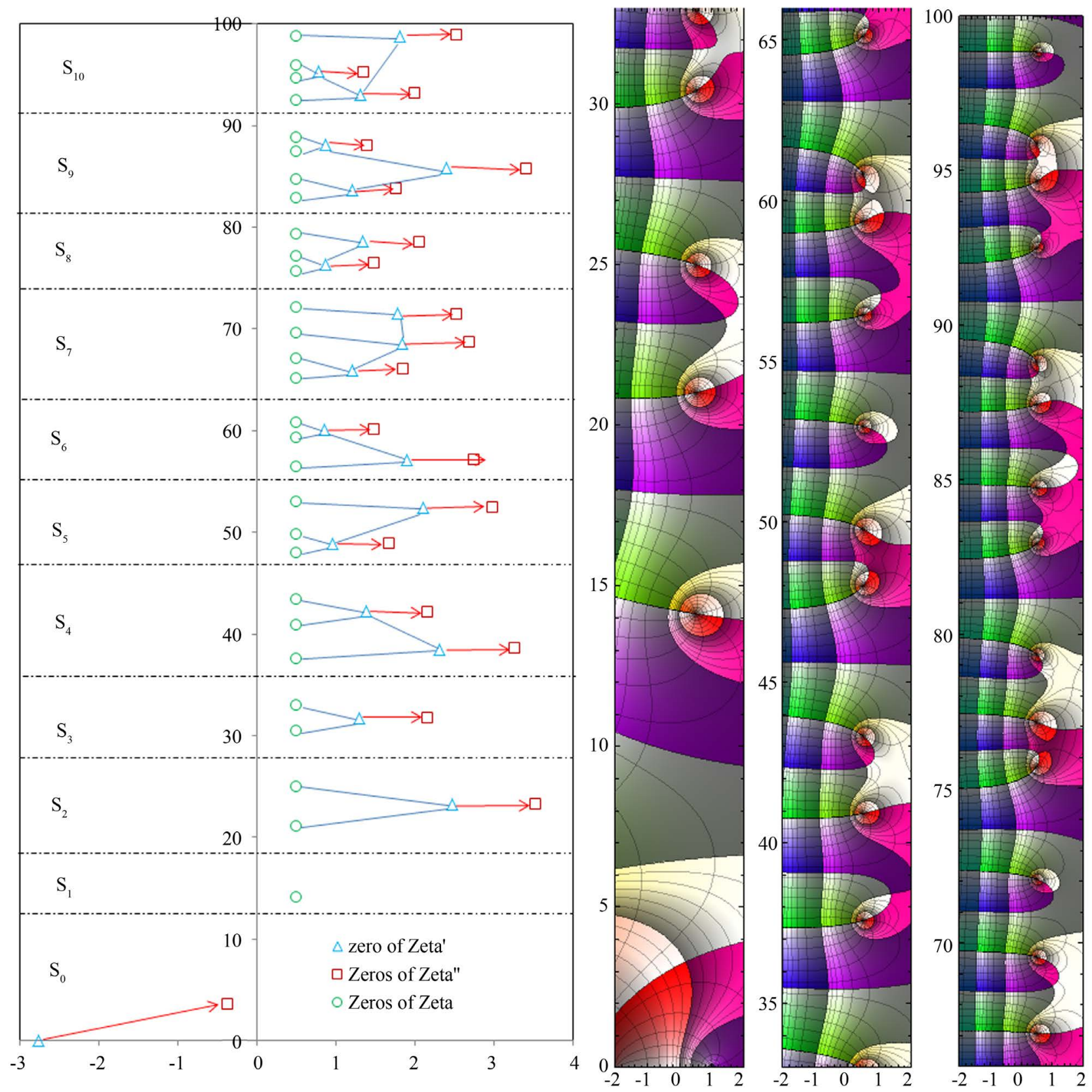

Figure 7. The zeros of $\zeta(s)$ and of its first two derivatives for $t \in[0,100]$.

are also clearly indicated. If there is a double zero of $\zeta_{A, \Lambda}(s)$ in $S_{k}$, then the respective leaf is counted twice as a leaf and once as an internal node, etc. When the number of zeros $s_{k, j}$ in $S_{k}$ is infinite we must have $\lim _{j \rightarrow \infty} s_{k, j}=\infty$, otherwise $s_{k, j}$ would have an accumulation point in $S_{k}$ and hence $\zeta_{A, \Lambda}(s)$ would be identically zero.

We can prove now also the Theorem 3.

Proof. Exactly 2 curves $\Gamma_{k, j}$ and $\Gamma_{k, j^{\prime}}$ should meet at a double zero $s_{0}$ of $\zeta_{A, \Lambda}(s)$ belonging to $S_{k}$. But $s_{0}$ is also a simple zero of $\zeta_{A, \Lambda}^{\prime}(s)$ and therefore a curve, say $\Upsilon_{k, j}$ should pass through $s_{0}$. Yet, $\Gamma_{k, j^{\prime}}$ intertwines with a curve $\Upsilon_{k, j^{\prime}}$ and unless this curve is $\Upsilon_{k, 0}$, which does not contain any 
zero of $\zeta_{A, \Lambda}^{\prime}(s)$, the point $s_{0}$ would be a double zero of for $\zeta_{A, \Lambda}^{\prime}(s)$, and this is impossible.

Hence, necessarily one of the curves $\Gamma_{k, j}$ passing through $s_{0}$ is $\Gamma_{k, 0}$. This shows that there can be only one double zero in $S_{k}$ and the other curve passing through $s_{0}$ in that case is either $\Gamma_{k, 1}$, or $\Gamma_{k,-1}$.

We notice that the curve $\Upsilon_{k, 0}$ does not contain any zero of $\zeta_{A, \Lambda}^{\prime}(s)$, hence it cannot pass through a multiple zero of order $m$ of $\zeta_{A, \Lambda}^{\prime}(s)$. Since every curve passing through that zero has an intertwining curve defined by the second derivative of $\zeta_{A, \Lambda}(s)$, the respective point should be a multiple zero of order $m$ of this second derivative, which is absurd. Therefore $\zeta_{A, \Lambda}^{\prime}(s)$ has no multiple zero and then $\zeta_{A, \Lambda}(s)$ cannot have any zero of the higher order than 2.

\section{Fundamental Domains}

For $j \neq 0$, the curves $\Gamma_{k, j}$ as well as $\Upsilon_{k, j}$ are parabola like curves with branches extending to infinity as $\sigma \rightarrow-\infty$. Therefore we can distinguish between the interior and the exterior of such curves. They can be viewed as oriented curves, with the same orientation as the real axis whose components of the pre-image they are. By the same rule $\Gamma_{k}^{\prime}$ and $\Upsilon_{k}^{\prime}$, as well as $\Upsilon_{k, 0}$ are also oriented, the positive orientation of $\Gamma_{k}^{\prime}$ and of $\Upsilon_{k, 0}$ being from the right to the left, while that of $\Upsilon_{k}^{\prime}$, is from the left to the right. Some curves $\Gamma_{k, j}, j \neq 0$ can contain in interior some other curves of the same type (embraced curves) and by the color alternating rule the orientation of the embracing curve and that of the embraced curves must be different. We did not find any instance where an embraced curve is in turn embracing, yet there is no reason to believe that such a situation is impossible.

Theorem 7 If the strip $S_{k}$ contains $m_{k}$ zeros counted with multiplicities, then $S_{k}$ can be partitioned into $m_{k}$ sub-strips which are fundamental domains for $\zeta_{A, \Lambda}(s)$

Proof. Suppose that curves $\Gamma_{k, j}$ and $\Gamma_{k, j^{\prime}}$ are containing the simple zeros $s_{k, j}$ and $s_{k, j^{\prime}}$ and two components of the pre-image of a circle $C_{r}$ which are going around each one of these zeros touch at a point $v_{k, j}$. This is a zero of $\zeta_{A, \Lambda}^{\prime}(s)$. The pre-image of the segment of line connecting $z=1$ and $\zeta_{A, \Lambda}\left(v_{k, j}\right)$ has as component an arc $\eta_{k, j}$ connecting the points on the two curves where $\zeta_{A, \Lambda}(s)=1$ and passing through $v_{k, j}$. If one of these curves is $\Gamma_{k, 0}$ then the respective point is $\infty$ and $\eta_{k, j}$ is an unbounded curve. The strip $\Omega_{k, j}$ bounded by this curve and the branches of $\Gamma_{k, j}$ and $\Gamma_{k, j^{\prime}}$ corresponding to the interval $[1,+\infty)$, turns out to be a fundamental domain of $\zeta_{A, \Lambda}(s)$. Indeed, $\Omega_{k, j}$ is mapped conformally by $\zeta_{A, \Lambda}(s)$ onto the whole complex plane with a slit alongside the interval $[1,+\infty)$ followed by a slit alongside the segment from $z=1$ to $\zeta_{A, \Lambda}\left(v_{k, j}\right)$. If $\Gamma_{k, j}$ embraces $\Gamma_{k, j^{\prime}}$ then $\Omega_{k, j}$ is bounded to the right.

Suppose now that one of the two zeros is a double zero of $\zeta_{A, \Lambda}(s)$. We know that $\Gamma_{k, 0}$ must pass through that zero and the part of $\Gamma_{k, 0}$ corresponding to 
the interval $[0,1]$ becomes part of the boundaries of adjacent fundamental domains. One of them will have as image the complex plane with a slit alongside the positive real half axis and for the other one a slit from $z=1$ to $z=\zeta_{A, \Lambda}\left(v_{k, j}\right)$ should be added, where $j$ is 1 or -1 .

We know that $s_{k, j}$ cannot have a higher order of multiplicity and therefore the cases analysed exhaust all the possibilities. Having in view Theorem 6, we conclude that the strip $S_{k}$ can be always divided into $m_{k}$ fundamental domains.

Figure 8 below illustrates this theorem for the case of the Riemann Zeta

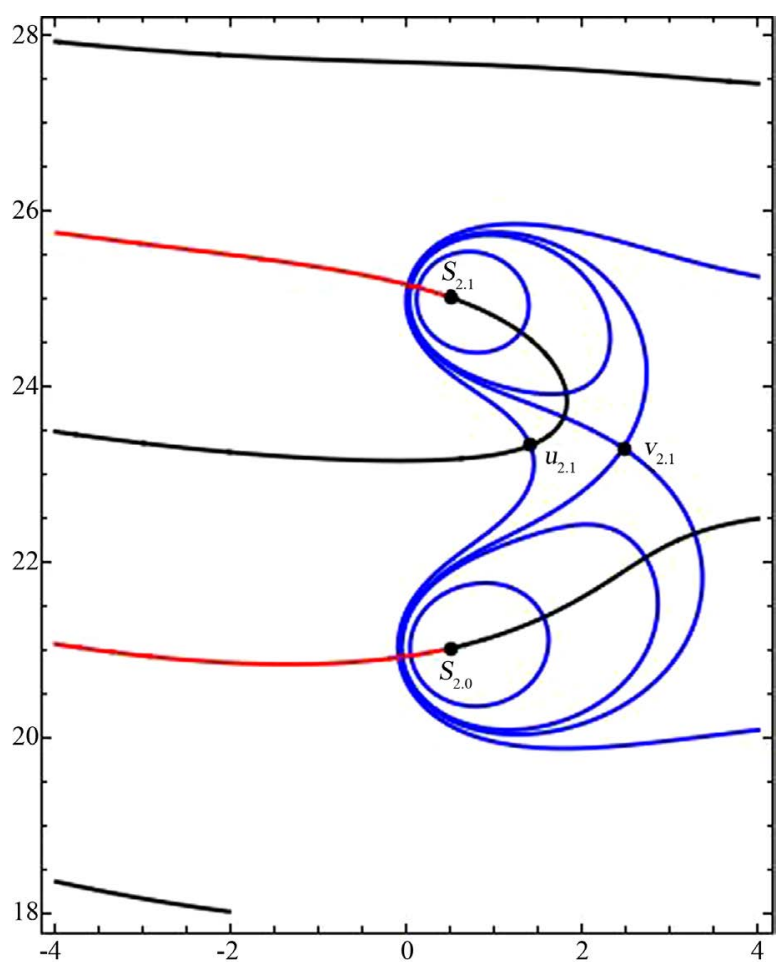

(a)

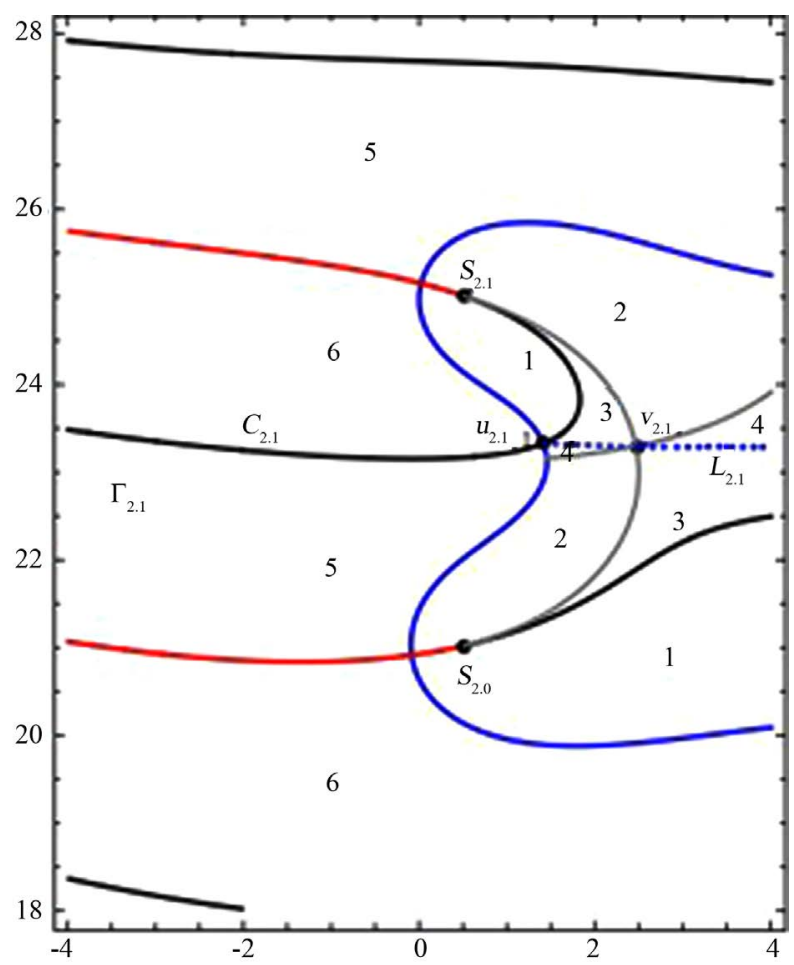

(b)

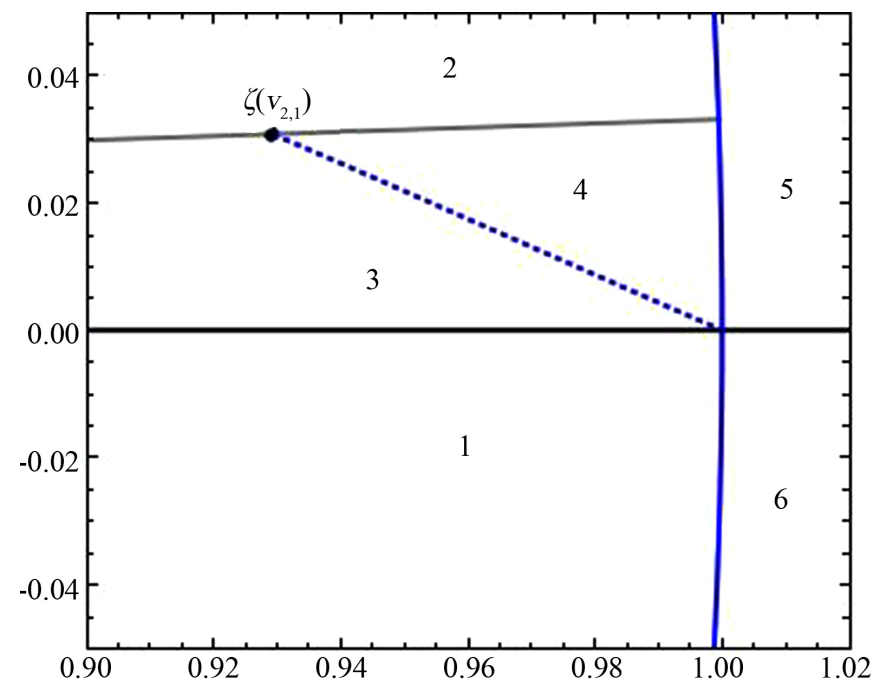

(c)

Figure 8. Two fundamental domains and their images. 
function and the strip $S_{2}$.

As seen in the case of Dirichlet L-functions (including the Riemann Zeta function) the strip $S_{0}$ has infinitely many zeros, yet following the same technique we can divide it into infinitely many fundamental domains.

If four different colors are used, say color 1 and 2 for the pre-images by $\zeta_{A, \Lambda}(s)$ of the positive and of the negative real half axis and 3 and 4 for the pre-images by $\zeta_{A, \Lambda}^{\prime}(s)$ of the same half axes, then two simple topological facts can be established [33]:

a) The color alternating rule, which states that as a point turns indefinitely in the same direction on a circle centred at the origin, the pre-images of this point by each one of the functions $\zeta_{A, \Lambda}(s)$ and $\zeta_{A, \Lambda}^{\prime}(s)$ will meet alternatively the colors 1 and 2, respectively 3 and 4 .

b) The color matching rule, which states that when intertwining curves meet each other, then if these are not $\Gamma_{k, 0}$ and $\Upsilon_{k, 0}$ color 1 will always meet color 4 and color 2 will always meet color 3 . Only the curves $\Gamma_{k, 0}$ and $\Upsilon_{k, 0}$ can intersect each other at points where color 2 can meet color 4 .

The series (1) which are Euler products display special important properties.

\section{Euler Products}

It is known that the Dirichlet L-functions are meromorphic continuations of ordinary Dirichlet series defined by Dirichlet characters and these series can be expressed as Euler products. This property is a corollary of the fact that the Dirichlet characters are totally multiplicative functions (see for example [33]. Yet the property of being total multiplicative can be extended to general Dirichlet series, as done in [35], and therefore some of the general Dirichlet series $\zeta_{A, \Lambda}(s)$ (see the details in [35]) can also be written as Euler products:

$$
\zeta_{A, \Lambda}(s)=\sum_{n=1}^{\infty} a_{n} \mathrm{e}^{-\lambda_{n} s}=\prod_{p \in \mathbb{P}}\left(1-a_{\mathrm{p}} \mathrm{e}^{-\lambda_{p} s}\right)^{-1},
$$

where $\mathbb{P}$ is the set of prime numbers. This convention will be kept in the following for all the products and sums involving the subscript $p$. The product has the same abscissa of convergence as the series itself.

Looking for counterexamples to the Grand Riemann Hypothesis (GRH), some Dirichlet series satisfying a Riemann type of functional equation have been found, whose analytic continuation exhibit off critical line non trivial zeros, namely the Davenport and Heilbronn type of functions and linear combinations of L-functions satisfying the same functional equation. Although these are not counterexamples to GRH, their study allowed us to draw interesting conclusions. We have seen in [36] that if $\zeta_{A, \Lambda}(s)$ does not satisfy the GRH, then for every two distinct non trivial zeros $s_{1}=\sigma+i$ and $s_{2}=1-\sigma+i t$ there is $\tau_{0}$, $0<\tau_{0}<1$ such that $\zeta_{A, \Lambda}^{\prime}\left(s\left(\tau_{0}\right)\right)=0$, where $s(\tau)=(1-\tau) s_{1}+\tau s_{2}$, i.e. the derivative of $\zeta_{A, \Lambda}(s)$ cancels at a point $s_{0}=s\left(\tau_{0}\right)$ of the interval $I$ determined by $s_{1}$ and $s_{2}$. Moreover, $\Re\left(s_{0}\right)<1 / 2$.

Let us rephrase and give a simplified proof to [36], Theorem 3.

Theorem 8 Suppose that the function (5) satisfies a Riemann type of func- 
tional equation and the respective series has the abscissa of convergence $\sigma_{c}<1 / 2$. Then for every non trivial zero $\sigma+i$ of $\zeta_{A, \Lambda}(s)$ we have $\sigma=1 / 2$.

Proof. Suppose that there is a zero $\sigma+i$ of $\zeta_{A, \Lambda}(s)$ for which $\sigma>1 / 2$. Then, due to the functional equation, $1-\sigma+i$ is also a zero of $\zeta_{A, \Lambda}(s)$ (see Figure 9 below). There is $r>0$ such that in the components $D_{1}$ and $D_{2}$ of the pre-image of the disc centred at the origin and of radius $r$ containing the respective zeros the function $\zeta_{A, \Lambda}(s)$ is injective. Then we can define the function $\phi: D_{1} \rightarrow D_{2}$ as follows:

$$
\phi(s)=\zeta_{A, \Lambda}(s)_{\mid D_{2}}^{-1} \circ \zeta_{A, \Lambda}(s)
$$

The function $\phi$ can be continued as an analytic involution of the union $\Omega \cup \Omega^{\prime}$ of the fundamental domains $\Omega$ and $\Omega^{\prime}$ of $\zeta_{A, \Lambda}(s)$ containing the respective zeros. The boundaries of the domains $\Omega$ and $\Omega^{\prime}$ have a common component $L$ and the union $\Omega \cup \Omega^{\prime} \cup L$ is a simply connected domain $H$. The function $\phi$ can be continued to an analytic involution of $H$ having $s_{0}$ as a fixed point. We have $\phi(\phi(s))=s$ in $H$, in particular $\phi(\sigma+i t)=1-\sigma+i t$ and $\phi(1-\sigma+i t)=\sigma+i t$. Moreover, $\zeta_{A, \Lambda}(\phi(s))=\zeta_{A, \Lambda}(s)$ in $H$. Let us define the function $\Phi$ by

$$
\Phi(s)=\zeta_{A, \Lambda}(s) / \zeta_{A, \Lambda}(\phi(s))
$$

Since the numerator and the denominator of $\Phi$ are analytic functions in $H$ and the denominator cancels only at $\sigma+i t$ and $1-\sigma+i t$, the function $\Phi$ is analytic in $H$ except at these two points. Since $\zeta_{A, \Lambda}(s)=\zeta_{A, \Lambda}(\phi(s))$, we have that $\Phi(s)=1$ for $s$ not equal to one of these points. Yet they are removable singularities, and we can set $\Phi(s)=1$ in $H$. By the formula (5) we have

$$
\Phi(s)=\Pi_{p \in \mathbb{P}}\left(1-a_{p} \mathrm{e}^{-\lambda_{p} \phi(s)}\right) /\left(1-a_{p} \mathrm{e}^{-\lambda_{p} s}\right)
$$

for $s \in H, \mathfrak{R}(s)>\sigma_{c}$. In particular,

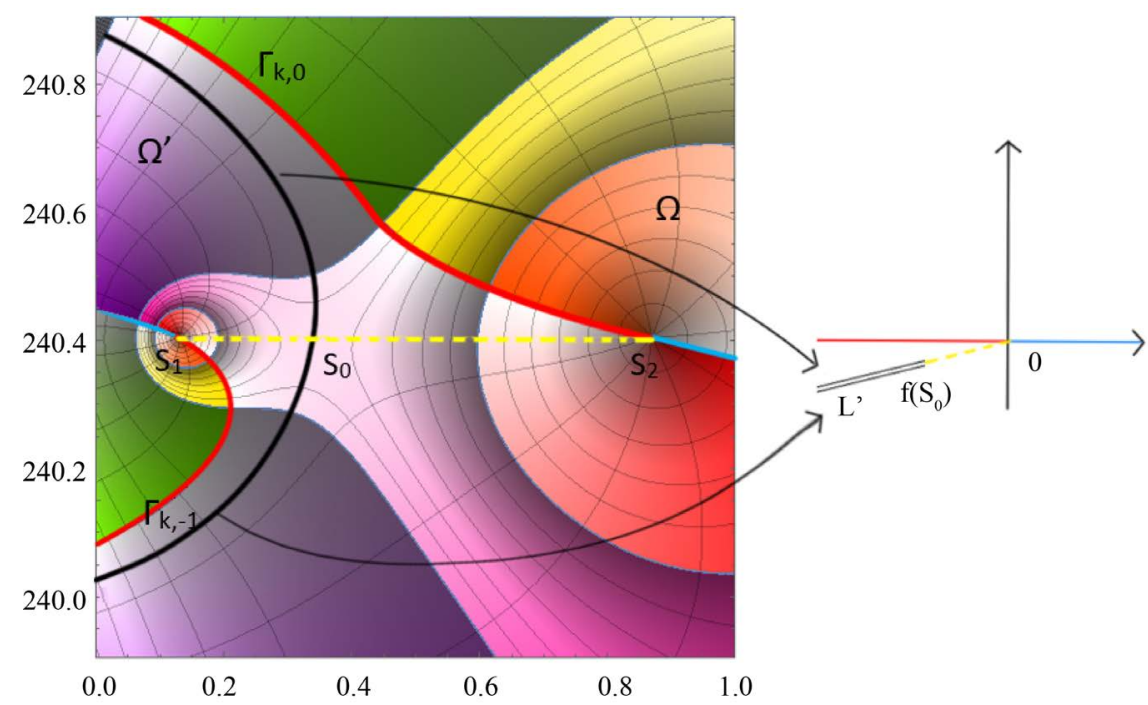

Figure 9. Symmetric zeros with respect to the critical line. 


$$
\begin{aligned}
1 & =\Phi(\sigma+i t) \\
& =\prod_{p \in \mathbb{P}}\left[1-a_{p} \mathrm{e}^{-\lambda_{p}(1-\sigma+i t)}\right] /\left[1-a_{p} \mathrm{e}^{-\lambda_{p}(\sigma+i t)}\right] \\
& =\Pi_{p \in \mathbb{P}}\left[e^{\lambda_{p} t i}-a_{p} \mathrm{e}^{-\lambda_{p}(1-\sigma)}\right] /\left[e^{\lambda_{p} t i}-a_{p} \mathrm{e}^{-\lambda_{p} \sigma}\right]
\end{aligned}
$$

The arguments of these ratios represent the angles under which the segment between $a_{p} \mathrm{e}^{-\lambda_{p}(1-\sigma)}$ and $a_{p} \mathrm{e}^{-\lambda_{p} \sigma}$ is seen from the point $\mathrm{e}^{\lambda_{p} t i}$ on the unit circle. If a Ramanujan type condition is fulfilled, namely $A$ and $\Lambda$ are such that for every $\epsilon>0$ we have $\lim _{n \rightarrow \infty}\left|a_{n}\right| \mathrm{e}^{-\lambda_{n} \varepsilon}=0$, then the respective angles tend to zero as $p \rightarrow \infty$. This appears to be a necessary condition for the convergence of the series.

$$
\sum_{p \in \mathbb{P}} \arg \left[\mathrm{e}^{\lambda_{p} t i}-a_{p} \mathrm{e}^{-\lambda_{p}(1-\sigma)}\right] /\left[\mathrm{e}^{\lambda_{p} t i}-a_{p} \mathrm{e}^{-\lambda_{p} \sigma}\right]
$$

However, the condition is implicitly satisfied since we know that $\Phi(s)$ is well defined in the domain $H$. The series is a continuous function of $\sigma$, yet it can take only integer multiple values of $2 \pi$, which is possible only if it is a constant. Since $\lim _{n \rightarrow \infty} a_{n} \mathrm{e}^{-\lambda_{n} \delta}=0$, the series can remain constant only if there is $p_{0}$ such that for $p>p_{0}$ we have $\arg \left[\mathrm{e}^{\lambda_{p} t i}-a_{p} \mathrm{e}^{-\lambda_{p}(1-\sigma)}\right] /\left[\mathrm{e}^{\lambda_{p} t i}-a_{p} \mathrm{e}^{-\lambda_{p} \sigma}\right]=0$. This can happen in two situations: either the three points $\mathrm{e}^{\lambda_{p} t i}, a_{p} \mathrm{e}^{-\lambda_{p} \sigma}$ and $a_{p} \mathrm{e}^{-\lambda_{p}(1-\sigma)}$ are collinear, or $a_{p} \mathrm{e}^{-\lambda_{p}(1-\sigma)}=a_{p} \mathrm{e}^{-\lambda_{p} \sigma}$ and in this last case $1-\sigma=\sigma$, i.e. $\sigma=1 / 2$. In the first case, a shift in $t$ will not affect the real part of the zeros, yet it will destroy the collinearity of the three points and therefore this situation can be ignored. The final conclusion is that $\varsigma_{A, \Lambda}(s)$ cannot have any non trivial zero $\sigma+i$ with $\sigma$ strictly greater than $1 / 2$. The zero $1 / 2+i t$ can be either a simple zero and then we have only one fundamental domain $\Omega$, or a double zero and then it is the fixed point of the involution $\phi(s)$. In both cases it is located on the critical line, which completely proves the theorem.

We need to point out the fact that Figure 9 above illustrates a situation in which the Equation (5) is not satisfied.

Remark: In all of our publications we understood by trivial zeros of an Lfunction those zeros which can be trivially computed. In this respect, the non trivial zeros of the alternating Zeta function $\zeta_{a}(s)=\left(1-2^{1-s}\right) \zeta(s)$ are the same as those of $\zeta(s)$ and $\zeta_{a}(s)$ satisfies the conditions of Theorem 8 , therefore its non trivial zeros are located on the critical line. Thus, $\mathrm{RH}$ is also true for $\zeta(s)$.

Similarly, the non trivial zeros of a Dirichlet L-function $L(q, 1, s)=\left(1-q^{1-s}\right) \zeta(s)$ defined by the principal character modulo $q$ are the non trivial zeros of the Riemann Zeta function.

Also, the non trivial zeros of a Dirichlet L-function induced by an imprimitive character are the non trivial zeros of the function defined by the associated primitive character. Consequently, the $\mathrm{RH}$ for any Dirichlet L-function is fulfilled. With this understanding of the concept of non trivial zeros, Theorem 8 represents the proof of GRH for a wide class of functions. 


\section{Acknowledgements}

The author is very much thankful to Florin Alan Muscutar for his contribution with computer generated graphics.

\section{References}

[1] Cahen, E. (1894) Sur la function $\zeta(s)$ de Riemann et sur des fonctions analogues, Ann. Ecole Norm., $3^{\text {e }}$ serie, t. 11.

[2] Cahen, E. (1918) Sur les séries de Dirichlet, Compte rendue, t. 166.

[3] Hadamard, J. (1896) Sur la distribution des zéros de la function $\zeta(s)$ de Riemann et ses cons équences arithmé tiques, Bull. Soc. math., t. 24.

[4] Hadamard, J. (1908) Sur les s éries de Dirichlet, Rendiconti di Palermo, t. 25.

[5] Landau, E. (1907) Über die Multiplication Dirichletscher Reihen, rendiconti di Palermo, t. 24.

[6] Landau, E. (1909) Über das Konvergenzproblem der Dirichletschen Reihen, Rendiconti di Palermo, t. 28.

[7] Landau, E. (1917) Neuer Beweis eines Haptsatzes aus der Theorie der Dirichletschen Reihen, Leipziger Ber., t. 69.

[8] Landau, E. (1921) Über die gleichmassige Konvergenz Dirichletscher Reihen, Math. Zeitschrift, t. 11.

[9] Bohr, H. (1913) Über die gleichmassige Konvergenz Dirichletscher Reihen, J. fuer Math, t. 143.

[10] Bohr, H. (1913) Einige Bemerkungen über das konvergenzproblem Dirichletscher Reihen, Rendicnti di Palermo, t. 37.

[11] Bohr, H. (1913) Darstellung der gleichmassigen Konvergenzabszisse einer Dirichletschen reihe $\sum_{\mathrm{n}=1}^{\infty} \frac{\mathrm{a}_{\mathrm{n}}}{\mathrm{n}^{\mathrm{s}}}$ als Funktionen der Koeffizienten der Reihe. Archiv der Mathematik und Physik, 21, 326-330.

[12] Bohr, H. (1913) Zür Theorie der allgemeinen Dirichletschen Reihen. Mathematische Annalen, 79, 136-156.

[13] Hardi, G.-H. (1911) The Multiplication of Dirichlet's Series. Vol. 10, London Mathematical Society, London.

[14] Hardy, G.H. and Riesz, M. (1915) The General Theory of Dirichlet's Series. Cambridge University Press, Cambridge.

[15] Kojima, T. (1914) On the Convergence Abscissa of General Dirichlet's Series. Tohoku Mathematical Journal, 6, 140-142.

[16] Kojima, T. (1916) Note on the Convergence Abscissa of Dirichlet's Series. Tohoku Mathematical Journal, 9, 28-37.

[17] Kuniyeda, M. (1916) Uniform Convergence Abscissa of General Dirichlet's Series. Tohoku J., t. 9, 7-27.

[18] Valiron, G. (1926) Théorie générale des séries de Dirichlet. Mémorial des sciences mathématiques, fascicule, 17, 1-56.

[19] Yu, J. (1963) Borel's Line of Entire Functions Represented by Laplace-Stieltjes Transformations. Acta Mathematica Sinica, 13, 471-484.

[20] Kong, Y.Y. and Daochun, S. (2008) On Type-Function and Growth of Laplace-Stieltjes Transformations Convergent in the Right Half Plane, 2008. Advances in Mathematics, 37, 197-205.

[21] Luo, X. and Kong, Y.Y. (2012) On the Order and Type of Laplace-Stieltjes Trans- 
forms of Slow Growth. Acta Mathematica Sinica, 32A, 601-607.

[22] Kong, Y.Y. and Yang, Y. (2014) On the Growth Properties of the Laplace-Stieltjes Transform. Complex Variables and Elliptic Equations, 59, 553-563. https://doi.org/10.1080/17476933.2013.766174

[23] Singhal, C. and Srivastava, G.S. (2015) On the Approximation of an Analytic Function Represented by Laplace-Stieltjes Transformation. Analysis in Theory and Applications, 31, 407-420.

[24] Srivastava, G.S. and Singhal, C. (2015) On the Generalized Order and the Generalized Type of Laplace-Stieltjes Transformation Convergent in the Right Half Plane. Global Journal of Pure and Applied Mathematics, 11, 469-477.

[25] Kamthan, P.K. and Shing Gautam, S.K. (1975) Bases in a Certain Space of Functions Analytic in the Half Plane. Indian Journal of Pure and Applied Mathematics, 6, 1066-1075.

[26] Khoi, L.H. (1998) Holomorphic Dirichlet Series in the Half Plane. Vietnam Journal of Mathematics, 26, 259-271.

[27] Defant, A., Garcia, D., Maestre, M. and Perez-Garcia, D. (2008) Bohr's Strip for Vector Valued Dirichlet Series. Mathematische Annalen, 342, 533-555. https://doi.org/10.1007/s00208-008-0246-Z

[28] Bonet, J. (2015) Abscissas of Weak Convergence of Vector Valued Dirichlet Series. arXiv: $1502.00418 \mathrm{v} 1$.

[29] Srivastava, B.L. (1983) A Study of Spaces of Certain Classes of Vector Valued Dirichlet Series. Thesis, Indian Institute of Technology Kanpur, Kalyanpur.

[30] Srivastava, G.S. and Sharma, A. (2011) Bases in the Space of Vector Valued Analytic Dirichlet Series. International Journal of Pure and Applied Mathematics, 7, 9931000.

[31] Srivastava, G.S. and Sharma, A. (2012) Spaces of Entire Functions Represented by Vector Valued Dirichlet Series of Slow Growth. Acta Universitatis Sapientiae, Mathematica, 4, 154-167.

[32] Sharma, A. and Srivastava, G.S. (2016) Coefficient Multipliers on Spaces of Vector Valued Entire Dirichlet Series.

[33] Ghisa, D. (2013) Fundamental Domains and the Riemann Hypothesis. Lambert Academic Publishing, Saarbrücken.

[34] Ghisa, D. (2014) On the Generalized Riemann Hypothesis, Complex Analysis and Potential Theory with Applications. Cambridge Scientific Publishers, Cambridge, 77-94.

[35] Ghisa, D. (2016) On the Generalized Riemann Hypothesis II. International Journal of Scientific and Innovative Mathematical Research, 4, 46-55.

[36] Ghisa, D. (2016) Fundamental Domains and Analytic Continuation of General Dirichlet Series. British Journal of Mathematics \& Computer Science, 9, 94-111.

[37] Andreian-Cazacu, C. and Ghisa, D. (2009) Global Mapping Properties of Analytic Functions. Proceedings of 7 th ISAAC Congress, London, 13-18 July 2009, 3-12.

[38] Andreian-Cazacu, C. and Ghisa, D. (2011) Fundamental Domains of Gamma and Zeta Functions. International Journal of Mathematics and Mathematical Sciences, 2011, Article ID: 985323. https://doi.org/10.1155/2011/985323

[39] Speiser, A. (1934) Geometrisches zür Riemannschen Zetafunction. Mathematische Annalen, 110, 514-521. https://doi.org/10.1007/BF01448042

[40] Arias-de-Reina, J. (2003) X-Ray of Riemann's Zeta-Function. ArXiv: math/0309433, $42 \mathrm{p}$. 
[41] Wegert, E. (2012) Visual Complex Functions. Springer, Basel. https://doi.org/10.1007/978-3-0348-0180-5

[42] Wegert, E. (2016) Visual Exploration of Complex Functions. In: Qian, T. and Rodino, L.G., Eds., Mathematical Analysis, Probability and Applications-Plenary Lectures, Vol. 177, Springer International Publishing, 253-279.

https://doi.org/10.1007/978-3-319-41945-9_10

[43] Barza, I., Ghisa, D. and Muscutar, F.A. (2014) On the Location of the Zeros of the Derivatives of Dirichlet L-Functions. Annals of the University of Bucharest (Mathematics Series), 5, 21-31.

[44] Cao-Huu, T., Ghisa, D. and Muscutar, F.A. (2016) Multiple Solutions of Riermann Type of Functional Equations. British Journal of Mathematics and Computer Science, 17, 1-12. https://doi.org/10.9734/BJMCS/2016/26322

Submit or recommend next manuscript to SCIRP and we will provide best service for you:

Accepting pre-submission inquiries through Email, Facebook, LinkedIn, Twitter, etc. A wide selection of journals (inclusive of 9 subjects, more than 200 journals)

Providing 24-hour high-quality service

User-friendly online submission system

Fair and swift peer-review system

Efficient typesetting and proofreading procedure

Display of the result of downloads and visits, as well as the number of cited articles

Maximum dissemination of your research work

Submit your manuscript at: http://papersubmission.scirp.org/

Or contact apm@scirp.org 